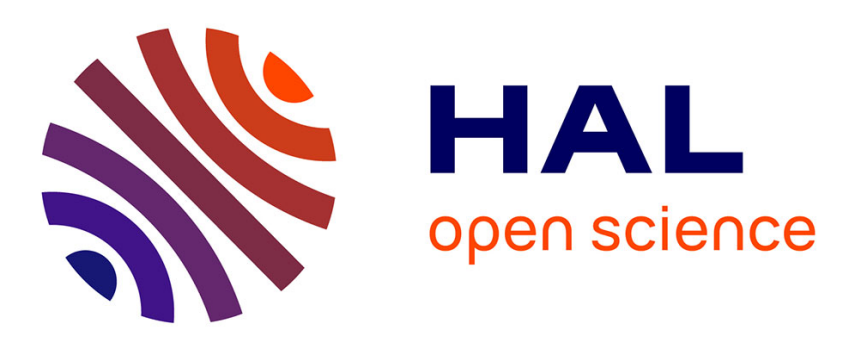

\title{
Likelihood of Meeting Isotropic Conditions in Reverberation Chambers from Power Transmission \\ Andrea Cozza
}

\section{To cite this version:}

Andrea Cozza. Likelihood of Meeting Isotropic Conditions in Reverberation Chambers from Power Transmission. IEEE Antennas and Wireless Propagation Letters, 2017, 16 (1), pp.2881-2884. 10.1109/LAWP.2017.2751103 . hal-01573543v2

\section{HAL Id: hal-01573543 \\ https://hal-centralesupelec.archives-ouvertes.fr/hal-01573543v2}

Submitted on 5 Dec 2017

HAL is a multi-disciplinary open access archive for the deposit and dissemination of scientific research documents, whether they are published or not. The documents may come from teaching and research institutions in France or abroad, or from public or private research centers.
L'archive ouverte pluridisciplinaire HAL, est destinée au dépôt et à la diffusion de documents scientifiques de niveau recherche, publiés ou non, émanant des établissements d'enseignement et de recherche français ou étrangers, des laboratoires publics ou privés. 


\title{
Likelihood of Meeting Isotropic Conditions in Reverberation Chambers from Power Transmission
}

\author{
Andrea Cozza, Senior Member, IEEE
}

\begin{abstract}
The ability of a metallic cavity to sustain an isotropic diffusive regime is a necessary condition in order to be used as a reverberation chamber ( $\mathrm{RC}$ ) in narrow-band settings, for which field samples are expected to behave as complex Gaussian random variables. Yet, no reliable method has been introduced so far that accurately predicts the lowest usable frequency (LUF), starting from which field samples are more likely to pass goodness-of-fit (GoF) tests, statistical tools that can measure how well field samples behave as Gaussian variables. This letter presents Monte Carlo simulation results that prove that the likelihood of passing GoF tests can be accurately predicted by measuring power transmission through the $\mathrm{RC}$, without any $a$ priori information about its characteristics. These results allow to identify the LUF from which a cavity is fit to generate rich multipath environments, as ideally expected for RCs, e.g., for over-the-air tests of wireless devices, and EMC tests.
\end{abstract}

Index Terms-Reverberation chamber, modal overlapping, Rayleigh fading, multipath propagation, statistical tests.

\section{INTRODUCTION}

Reverberation chambers (RCs) are test facilities that are expected to generate a rich multi-path environment, which can be described by means of a superposition of $N$ plane waves,

$$
\boldsymbol{E}(\boldsymbol{r}, \nu)=\sum_{n=1}^{N(\nu)} \tilde{\boldsymbol{E}}\left(\hat{\boldsymbol{k}}_{n}(\nu)\right) \mathrm{e}^{-\mathrm{j} k_{o} \hat{\boldsymbol{k}}_{n}(\nu) \cdot \boldsymbol{r}}
$$

where $\hat{\boldsymbol{k}}_{n}(\nu)$ and $\tilde{\boldsymbol{E}}\left(\hat{\boldsymbol{k}}_{n}(\nu)\right)$ are, respectively, the direction of arrival and the complex amplitude of the $n$th plane wave, both functions of frequency $\nu ; r$ is the position of the observer and $k_{o}=2 \pi / \lambda$ the wavenumber associated to the wavelength $\lambda$. Under ideal conditions, $N \rightarrow \infty$ and energy injected into the $\mathrm{RC}$ is scattered with equal probability among all possible states, i.e., angles of arrival, polarization and amplitude, a propagation regime implying isotropic diffusion [1], [2], which results into a homogeneous spatial distribution of energy. This condition makes up the basis for the ideal ability of RCs to yield position- and orientation-independent test results [3].

It is therefore of practical importance to know the lowest usable frequency (LUF) from which an RC approximates with sufficient accuracy isotropic conditions. RC users may rely on the use of goodness-of-fit (GoF) tests [4], to evaluate the likelihood that a population of samples adhere to a reference probability law: in fact, ideal diffusion leads to Gaussiandistributed field samples [3]. This approach necessarily implies an a posteriori decision, since based on measured samples, with no possibility of predicting the LUF [5], [6].

A. Cozza is with PIEM, GeePs (UMR 8507) CNRS - CentraleSupelec Univ Paris Sud - UPMC, 11 rue Joliot-Curie, 91192 Gif-sur-Yvette, France. Contact e-mail: andrea.cozzalieee.org.
As opposed to plane-wave models, as in (1), preferred in communication theory, physical considerations rather lead to a different representation [1], seeing the field distribution within a metallic cavity as the result of a superposition of independent resonant responses, or modes, i.e.,

$$
\boldsymbol{E}(\boldsymbol{r}, \nu)=\sum_{i \in \mathscr{I}} \gamma_{i} \boldsymbol{e}_{i}(\boldsymbol{r}) \psi_{i}(\nu)
$$

where $\boldsymbol{e}_{i}(\boldsymbol{r})$ is the spatial distribution of the standing wave underpinning the $i$ th mode, $\psi_{i}(\nu)$ its Lorentzian frequency response and $\gamma_{i}$ its complex amplitude. When used in a narrow-band setting, only the few modes with a resonance frequency close to the working frequency contribute [7]: these modes are collected into the set of indexes $\mathscr{I}$.

Based on (2), a number of criteria have been proposed to identify diffusive conditions, all of them requiring a minimum number of degrees of freedom, here modes: either looking for modal density [3], modal overlap [7] or, similarly, minimum electrical dimensions, often set with respect to the first $\mathrm{RC}$ resonance [8]. While most of these criteria are based on rules of thumb, none of them provides any quantitative estimate of how likely GoF tests would be passed.

This paper fills in this gap, by proving that there exists a direct relationship between average power transmission through an $\mathrm{RC}$ and probability of rejecting the hypothesis of isotropic diffusion. All predictions are supported by experimental results. This work is expected to help RC users to more easily and confidently predict over what frequency range isotropic diffusion can be obtained with sufficient accuracy, by means of a straightforward measurement procedure.

\section{PRobABILITY OF REJECTION FROM MODAL OVERLAP}

Eq. (2) can be implemented into a Monte Carlo (MC) scheme, in order to generate large populations of random field samples sharing the same average number of overlapping modes. This pool of data can then be used in order to explore the relationship between modal overlap and the degree of diffusion.

Field samples are generated at an arbitrary position $\boldsymbol{r}$, taking a single scalar component of the electric field along a direction $\hat{\boldsymbol{p}}$, as a linearly polarized antenna or probe would do. Hence, (2) reduces to $\hat{\boldsymbol{p}} \cdot \boldsymbol{E}(\boldsymbol{r}, \nu)=\sum_{i} \gamma_{i}^{\prime}(\boldsymbol{r}) \psi_{i}(\nu)$, with $\gamma_{i}^{\prime}$ now accounting for the randomness of spatial modal distributions and their excitation coefficients. In the MC scheme, the $\left\{\gamma_{i}^{\prime}\right\}$ were modelled as zero-mean Gaussian random variables, while the frequencies of resonance involved in the $\left\{\psi_{i}(\nu)\right\}$ were considered as uniformly distributed around the working frequency. Defining as $B_{m}$ the $3 \mathrm{~dB}$ modal bandwidth covered by $\psi_{i}(\nu)$, an average modal overlap $M_{W}$ (see sec. III) is enforced by 


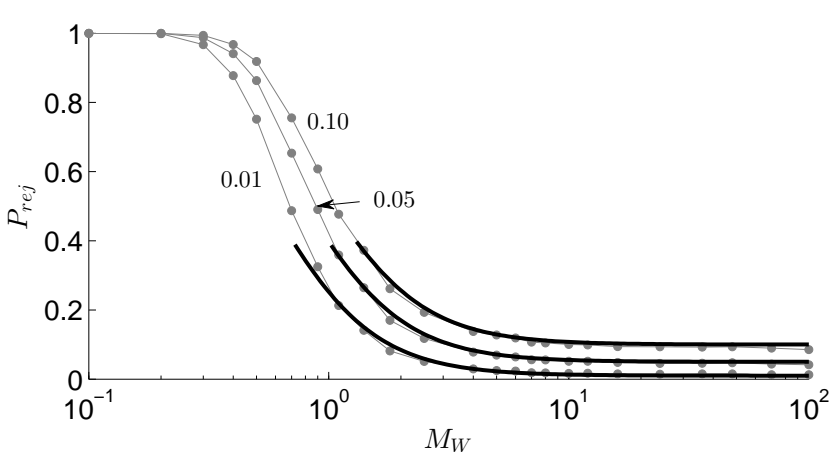

Fig. 1: Monte Carlo estimated probability of rejection from KS tests of 100-sample data sets, as a function of the average modal overlap $M_{W}$ and significance level $\alpha$ (marked values) for the null hypothesis of isotropic diffusion. Solid lines represent approximation (3).

distributing $N_{M}=M_{W} B_{e} / B_{m}$ resonant frequencies over a bandwidth $B_{e}$. In this paper $B_{e} / B_{m}=50$, in order to enable $M_{W}<1$ to be simulated, since modes come as integers.

This protocol was used in order to generate sets of $10^{5}$ field samples for each value of $M_{W}$ going from 0.1 to 100 . From this pool of data, subsets of $N_{s}$ samples were extracted randomly, in order to study the impact of sample population size, namely for 50,100, 250, 500 and 1000 samples. This choice covers most of the practical cases encountered by RC users. For each population size, 1000 random realizations were created in this way, practically independent because of the shear number of available combinations.

As recalled when introducing (1), isotropic diffusion implies that scalar components of field samples accurately follow a complex Gaussian distribution, or exponential when dealing with quantities proportional to their squared absolute value, as is received power. These expectations are never fully met, as they would require an infinite number of degrees of freedom. But they can still be acceptable in statistical terms, since the underlying distribution of populations of finite size can only be inferred with finite precision. GoF tests take this statistical uncertainty into account, by defining a statistics (i.e., a metric), based on the available samples, whose distribution is known in case field samples follow, e.g., an ideal Gaussian distribution: this is known as the null hypothesis $H_{o}$.

In this respect, Kolmogorov-Smirnov (KS) test was applied on the MC data subsets, but other tests may also be used without any change in the procedure. KS test is based on the statistics $D_{\mathrm{KS}}=\max _{x}|\hat{F}(x)-F(x)|$, with $\hat{F}(x)$ the empirical cumulative distribution obtained from the available subset of data and $F(x)$ the reference distribution associated to the null hypothesis $H_{o}$ of isotropic diffusion. $D_{\mathrm{KS}}$ is expected to be smaller than a critical value $D_{\mathrm{cr}}=D_{\alpha} / \sqrt{N_{s}}$ with a probability $1-\alpha$, where $\alpha$ represents the probability of rejecting $H_{o}$ even when the samples are distributed according to $H_{o}$, i.e., a false alarm: typical values of $\alpha$ are chosen around 0.05 . According to the measurement of power transmission discussed in sec. III, an exponential distribution is expected for ideal conditions. Then $D_{\alpha}=1.25,1.06$ and 0.96 , for

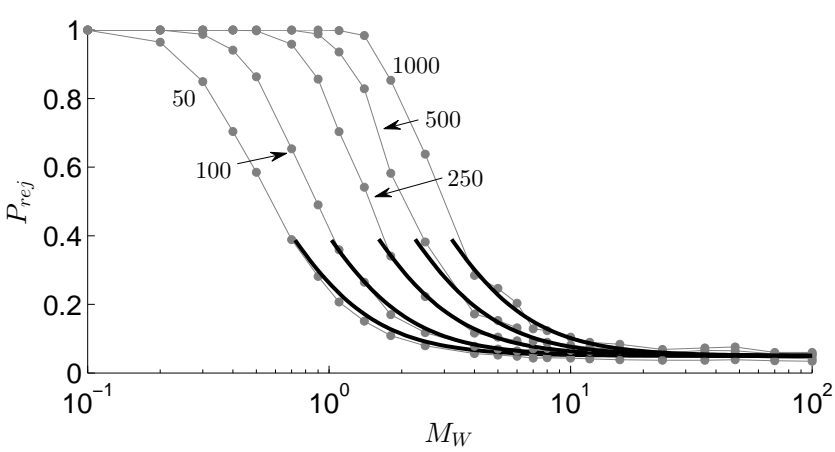

Fig. 2: Monte Carlo estimated probability of rejection from KS tests for a significance level $\alpha=0.05$, as a function of the average modal overlap $M_{W}$ and the size of the data sets (marked values). Solid lines represent approximation (3).

$\alpha=0.01,0.05$ and 0.1 , respectively. These values are those for a Lilliefors test, taking into account the fact that the exponential distribution requires one parameter (its average) to be empirically estimated [9].

For ideal conditions the probability $P_{\text {rej }}$ of observing $D_{\mathrm{KS}}>D_{\text {cr }}$ coincides with the significance level $\alpha$; otherwise, a higher value would be expected, pointing to a measurable departure from $H_{o} . P_{\text {rej }}$ can be estimated from the set of $D_{\mathrm{KS}}$ obtained for each random realization of samples generated by the MC scheme, by computing the empirical cumulative distribution function of $D_{\mathrm{KS}}$ and thus the probability that $D_{\mathrm{KS}}>D_{\mathrm{cr}}$.

Figs. 1 and 2 show how $P_{\text {rej }}$ evolves as $N_{s}$ and $M_{W}$ change, for different values of $\alpha$. It can be noticed how a transition occurs as $M_{W} \gtrsim 1$. The transition region changes with $N_{s}$, since as it increases GoF tests become more selective at detecting ever smaller drifts from $H_{o}$. These results unambiguously prove that $P_{\text {rej }}$ is univocally determined by $M_{W}$ and $N_{s}$, so that knowledge of $M_{W}$ would allow predicting the likelihood of an RC performance undistinguishable from ideal isotropic diffusion. $P_{\text {rej }}$ decreases monotonously as $M_{W}$ increases, converging to $P_{\text {rej }}=\alpha$ as $M_{W} \rightarrow \infty$. Therefore, there exists a critical $M_{W}$ representing the minimum number of effective degrees of freedom needed in order to ensure that $P_{\text {rej }}$ be small enough.

In order to provide for simple decision criteria, the following approximation of the MC results was studied

$$
P_{\mathrm{rej}}\left(M_{W}, \alpha, N_{s}\right) \simeq \frac{1-\alpha}{1+\left(M_{W} / M_{c}\right)^{2}}+\alpha,
$$

where $M_{c}=M_{\alpha} \sqrt{N_{s}}$ is a characteristic modal overlap, for which $P_{\text {rej }} \simeq 0.5$. $M_{\alpha}$ was found only to depend on $\alpha$, and equal to $0.0917,0.0759$ and 0.0569 for $\alpha=0.01,0.05$ and 0.1 , respectively. Approximation (3) only holds for $P_{\text {rej }} \lesssim 0.4$ and covers the region of decision values used in practice. Its accuracy is shown in Figs. 1 and 2.

From (3), a decision criterion ensues

$$
M_{W} / M_{c} \geqslant \sqrt{\frac{1-\left(P_{\mathrm{th}}-\alpha\right)}{P_{\mathrm{th}}-\alpha}}
$$


where $P_{\mathrm{th}}$ is the maximum probability of rejection accepted by an RC user. For a significant increase in $P_{\text {rej }}$, e.g., $P_{\text {th }}-\alpha \leqslant$ 0.1 , (4) yields $M_{W} / M_{c} \geqslant 3$.

\section{MODAL OVERLAP FROM POWER TRANSMISSION}

Recalling that the modal bandwidth $B_{m}(\nu)=\nu / Q(\nu)$, with $Q(\nu)$ the average quality factor of the RC, its average modal overlap is $M_{W}(\nu)=m_{W}(\nu) \nu / Q(\nu)$, where the average modal density $m_{W}(\nu)$ of an electromagnetic cavity of volume $V$ was predicted by $\mathrm{H}$. Weyl to be $m_{W}(\nu)=8 \pi V \nu^{2} / c^{3}$, with $c$ the speed of light for the medium filling the cavity. Hence,

$$
M_{W}(\nu)=8 \pi V_{\lambda}(\nu) / Q(\nu)
$$

with $V_{\lambda}(\nu)=V / \lambda^{3}$.

Several methods have been developed in order to estimate $Q(\nu)$, of which the most popular is based on the measurement of power transmission through the RC [3]. The method requires two antennas to be present within the $\mathrm{RC}$ and to measure the average power transmission. When done by means of a vector network analyzer (VNA), $S_{i j}$ scattering parameters are measured for each random realization generated by a stirring technique, e.g., a rotating paddle. The internal power transmission, i.e., the fraction of power transmission occurring within the RC, is then estimated

$$
T(\nu)=\frac{\left\langle\left|S_{21}\right|^{2}\right\rangle}{\eta_{1} \eta_{2}\left(1-\left|\Gamma_{1}\right|^{2}\right)\left(1-\left|\Gamma_{2}\right|^{2}\right)}
$$

where $\langle\cdot\rangle$ is the ensemble average, inevitably substituted by the sample mean during experiments. $\Gamma_{i}$ is the reflection coefficient of the $i$ th antenna, either measured in free-space conditions or estimated from RC measurements of the $S_{i i}$ scattering parameter, as $\Gamma_{i} \simeq\left\langle S_{i i}\right\rangle$. The radiation efficiency of the antenna, $\eta_{i}$, also needs to be taken into account, in order to estimate the actual power transmitted through the RC.

It can then be demonstrated that [3]

$$
T(\nu)=Q(\nu) / Q_{a}(\nu),
$$

with $Q_{a}(\nu)=16 \pi^{2} V_{\lambda}$ the contribution of antenna coupling to power lost by the RC [3]. This definition does not consider non-idealities in the antennas, as they are already taken into account in (6).

From (5) and (7), it is therefore possible to directly express

$$
\hat{M}_{W}(\nu)=1 / 2 \pi T(\nu),
$$

where the hat identifies an estimator, as opposed to $M_{W}$ enforced during the $\mathrm{MC}$ simulations. The above expression makes $M_{W}(\nu)$ more tangible, as it now directly appears to be related to power transmission, a quantity that can be estimated and even monitored quite easily, without a priori information about the RC.

Alternatively, a similar result can also be derived in case power reflection rather than transmission is measured. Estimating $Q(\nu)$ from $S_{i i}$ data has been discussed in [10]. In this case, instead of (6), $R(\nu)$ should be used, defined as

$$
R(\nu)=\frac{\left\langle\left|S_{i i}\right|^{2}\right\rangle-\left|\Gamma_{i}\right|^{2}}{\eta_{i}^{2}\left(1-\left|\Gamma_{i}\right|^{2}\right)^{2}},
$$

which was shown to be related to (6) as $R(\nu)=2 T(\nu)$ [11], thus yielding $\hat{M}_{W}(\nu)=1 / \pi R(\nu)$.

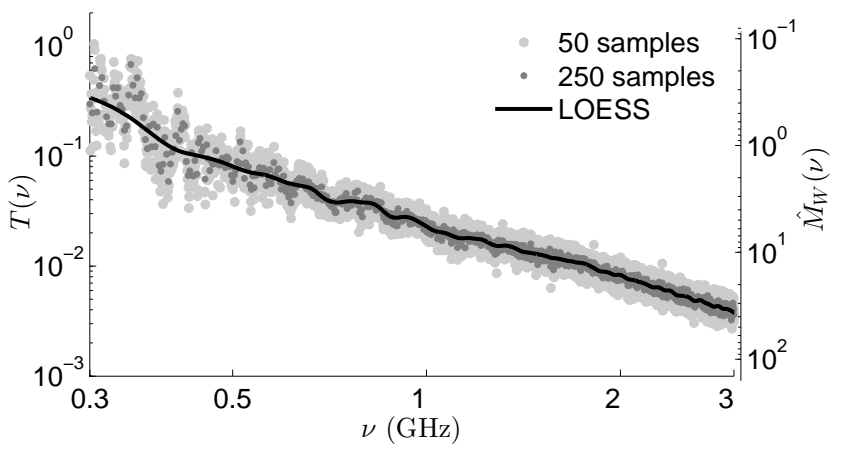

Fig. 3: Internal power transmission estimated from measured data and their LOESS-smoothed version obtained over 300 $\left(N_{s}=50\right)$ and $60\left(N_{s}=250\right)$ adjacent frequency samples (thick solid line). The modal overlap expected from (8) is shown on the right-hand vertical axis.

\section{EXPERIMENTAL VALIDATION}

The accuracy of these results and predictions were tested in a cuboid RC, with dimensions $5.95 \times 2.46 \times 3.24 \mathrm{~m}^{3}$ in CentraleSupelec. Two sphere-capped monocone antennas were placed within the RC, oriented in such a way as to have their axis orthogonal, thus minimizing any direct coupling. Weakly-directive antennas allow to excite the largest number of directions of arrival in the RC. These antennas are made of copper and not electrically small, being about $\lambda / 5$ high at $300 \mathrm{MHz}$; their radiation efficiency can therefore be assumed to be close to one. The antennas were connected to a VNA outside the RC, with cables whose losses were fully calibrated and compensated. A Z-folded stirrer was oriented along 50 uniformly spaced positions, in order to introduce randomness in the transmission process. 6000 samples of the three main scattering parameters were measured over the frequency range 0.3 to $3 \mathrm{GHz}$, for each stirrer position, and translated into an estimate of $T(\nu)$, as defined in (6). The background lag-1 auto-correlation between the samples was found to be lower than $5 \%$ over the entire frequency range.

$T(\nu)$ was then smoothed using a local regression (LOESS) processing, in order to reduce the impact of outliers at low frequency and the uncertainty due to the limited number of available samples. Results are in Fig. 3. From the same data KS statistics were computed for each frequency, yielding one $D_{\mathrm{KS}}(\nu)$ value for each population. In order to estimate the probability of rejection $P_{\text {rej }}(\nu)$, the distribution function of KS was estimated by clustering 50 neighboring frequencies on a receding-horizon basis, finally computing the probability that $D_{\mathrm{KS}}(\nu)>D_{\mathrm{cr}}$, as discussed in sec. II. In order to test the impact of increasing the population size $N_{s}$, raw measured data were clustered over 2 or 5 adjacent frequencies, yielding 100- and 250-sample populations; the processing for the computation of $P_{\text {rej }}(\nu)$ is the same, apart for $D_{\text {cr }}$, which changes according to $N_{s}$. The results of this operation are shown in Fig. 4.

These empirical results are expressed as functions of $T(\nu)$ in Fig. 5, where they are compared to MC results using (8) and LOESS results in Fig. 3, in order to map $M_{W}(\nu) \mapsto T(\nu)$. 


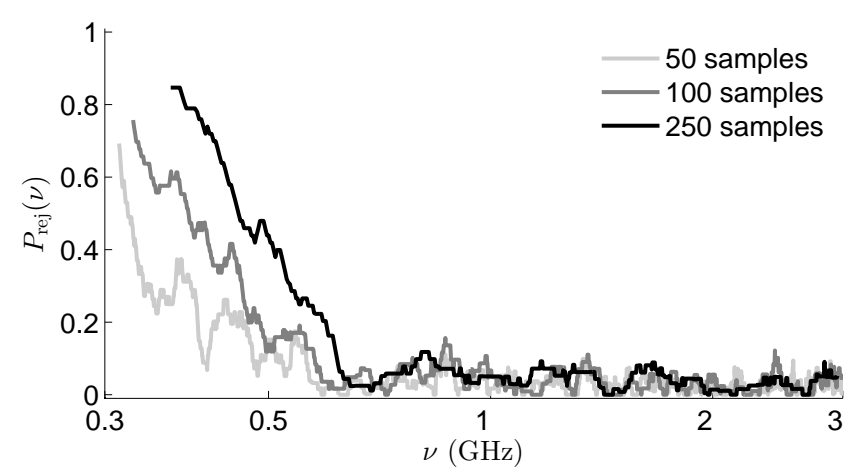

Fig. 4: Probability of rejecting $H_{o}$ (ideal isotropic conditions) estimated from experimental data, for three sample population sizes.

Fig. 5 confirms the validity of the MC results and the fact that $T(\nu)$ gives access to an accurate estimator of $P_{\text {rej }}(\nu)$.

In particular, (3) and (8) can be used for predicting the maximum $T(\nu)$ such that $P_{\text {rej }}(\nu) \leqslant P_{\text {th }}$. E.g., for $P_{\text {th }}-\alpha<0.1$ and $\alpha=0.05$, (4) and (8) require $T(\nu) \leqslant 1 / 1.43 \sqrt{N_{s}}$, hence $T(\nu) \leqslant 0.099,0.069$ and 0.044 , corresponding to $M_{W}(\nu) \geqslant 1.6,2.3$ and 3.6, for $N_{s}=50,100$ and 250, respectively. From Fig. 3, the LUFs are therefore expected to be found around 447, 539 and $683 \mathrm{MHz}$, which are within less than $10 \%$ of the frequencies at which data in Fig. 4 cross $P_{\text {th }}=0.15$.

In practice, using GoF on empirical data does not allow a clean-cut decision criterion, because of the inevitable statistical uncertainty with which $P_{\text {rej }}(\nu)$ is evaluated, clearly observable in Fig. 4. Fig. 5 shows that the fluctuations in $P_{\text {rej }}(\nu)$ are indeed well explained by using a binomial law applied to MCpredicted values of $P_{\text {rej }}$, modeling the uncertainty inherent in empirical frequencies of occurrence. Another advantage of results in Figs. 1 and 2, together with their approximation (3), is that they do not suffer from significant statistical uncertainty.

\section{CONCLUSions}

MC simulations have demonstrated the univocal relationship existing between power transmission $T(\nu)$ and the likelihood of measuring field samples closely approximating the behavior of a perfectly isotropic diffusion within an RC. Given the maximum acceptable risk of finding an RC not performing as expected, the results presented in this paper accurately predict the LUF from which an RC can be safely operated. Thanks to its intrinsically smooth and regular nature [3], $T(\nu)$ can be estimated even from relatively small sets of samples, using smoothing techniques, as LOESS, applied across frequency.

The fact that the proposed models, when applied to empirical estimates of the modal overlap $\hat{M}_{W}$, accurately predict the probability of rejection, is an important result in its own right, since it provides unequivocal evidence that modal overlap estimators accurately represent the number of effective degrees of freedom underpinning the behavior of an RC. Moreover, $M_{W}(\nu)$ is more readily accessible than the number of plane waves $N(\nu)$, thus making it a natural candidate for predicting the stochastic performance of an RC.

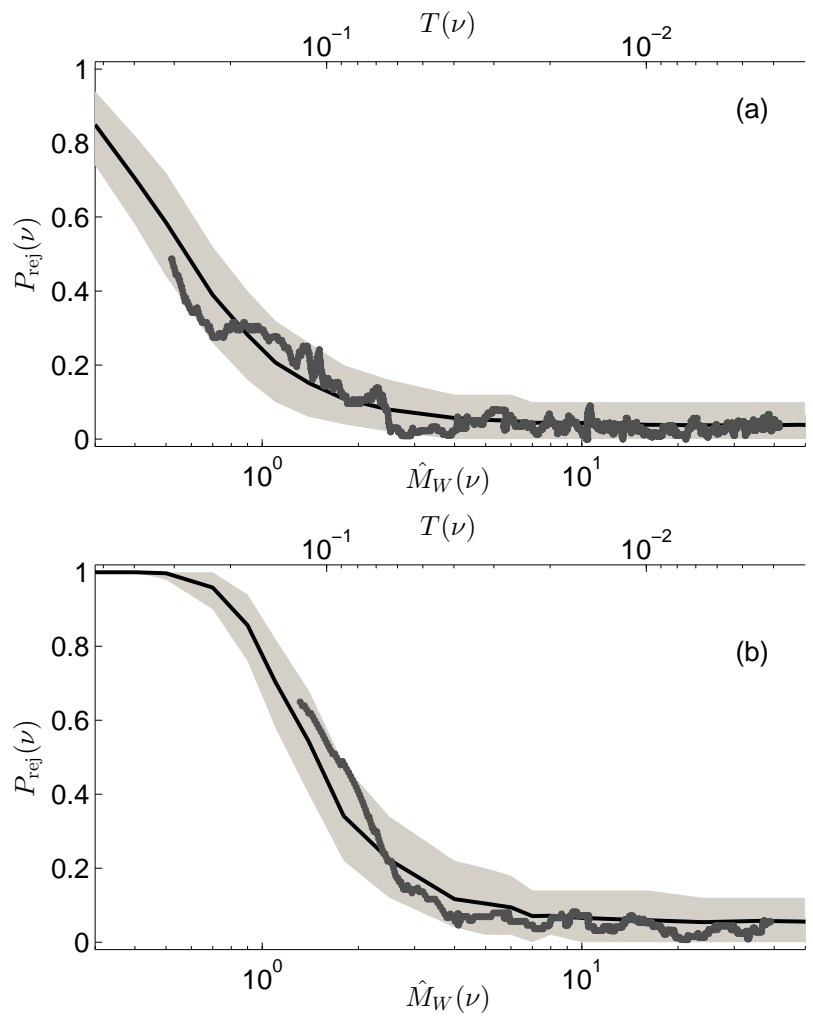

Fig. 5: Probability of rejecting $H_{o}$ expressed as a function of power transmission $T(\nu)$ (top axis) and estimated modal overlap $\hat{M}_{W}(\nu)$ (bottom axis), for $N_{s}$ equal to: (a) 50 and (b) 250. The solid thick lines correspond to the MC-based results results in Fig. 2, with the shaded area marking the $95 \%$ confidence interval of estimators of $P_{\text {rej }}$ (binomial process).

\section{REFERENCES}

[1] P. Morse and H. Feshbach, Methods of Theoretical Physics. Feshback Publishing, 1981.

[2] A. Ishimaru, Wave propagation and scattering in random media. WileyIEEE Press, 1999, vol. 12.

[3] D. Hill, "Electromagnetic Theory of Reverberation Chambers," National Institute of Standards and Technology, Tech. Rep. 1506, 1998.

[4] R. D'Agostino and M. Stephens, Goodness-of-fit techniques. CRC, 1986.

[5] C. Lemoine, P. Besnier, and M. Drissi, "Investigation of reverberation chamber measurements through high-power goodness-of-fit tests," IEEE Trans. Electromagn. Compat., vol. 49, no. 4, pp. $745-755$, nov. 2007.

[6] A. Adardour, G. Andrieu, and A. Reineix, "On the low-frequency optimization of reverberation chambers," IEEE Trans. Electromagn. Compat., vol. 56, no. 2, pp. 266-275, April 2014.

[7] A. Cozza, "The role of losses in the definition of the overmoded condition for reverberation chambers and their statistics," IEEE Trans. Electromagn. Compat., vol. 53, no. 2, pp. 296-307, May 2011.

[8] Reverberation chamber test methods, International Electrotechnical Commission (IEC) Std. 61 000-4-21, 2003.

[9] H. L. Crutcher, "A note on the possible misuse of the KolmogorovSmirnov test," Journal of Applied Meteorology, vol. 14, no. 8, pp. 16001603, 1975.

[10] P. Besnier, J. Sol, A. Presse, C. Lemoine, and A. C. Tarot, "Antenna efficiency measurement from quality factor estimation in reverberation chamber," in 46th European Microwave Conference (EuMC), Oct 2016, pp. $715-718$.

[11] I. Junqua, P. Degauque, M. Liénard, and F. Issac, "On the power dissipated by an antenna in transmit mode or in receive mode in a reverberation chamber," IEEE Trans. Electromagn. Compat., vol. 54, no. 1, pp. 174-180, Feb 2012. 